\title{
Modeling the Time Varying Behavior of Mobile Ad-Hoc Networks
}

\author{
David Tipper \\ University of Pittsburgh \\ 135 N. Bellefield Avenue \\ Pittsburgh, PA USA 15260 \\ 1 (412) 624-9421 \\ tipper@tele.pitt.edu
}

\author{
Yi Qian \\ University of Puerto Rico at Mayaguez \\ P.O. Box 9080 \\ Mayaguez, PR 00681 \\ 1 (787) 458-4901 \\ yqian@ece.uprm.edu
}

\author{
Xiaobing Hou \\ University of Pittsburgh \\ 135 N. Bellefield Avenue \\ Pittsburgh, PA USA 15260 \\ 1 (412) 624-9421 \\ xiaobing@mail.sis.pitt.edu
}

\begin{abstract}
In this paper we develop a performance modeling technique for analyzing the time varying performance of mobile ad hoc networks. Our approach is a novel hybrid of discrete event simulation and numerical method based queueing analysis. Network queues are modeled using fluid-flow based differential equation models which are solved using numerical methods, while node mobility is modeled using an adjacency matrix topology representation whose values are determined via discrete event simulation techniques. Numerical results are given illustrating the approach.
\end{abstract}

\section{Categories and Subject Descriptors}

C. Computer Systems Organization, C.4 Performance of Systems, Subjects: Modeling Techniques

\section{General Terms}

Algorithms, Performance, Reliability

\section{Keywords}

Ad-hoc networks, performance evaluation and modeling, time varying behavior

\section{INTRODUCTION}

Mobile ad-hoc networks using wireless links are expected to become an important part of the communication infrastructure. In a mobile ad-hoc network, nodes must cooperate to dynamically establish routes using multihop wireless links. There is no stationary infrastructure, and each node acts as a router. A packet may have to be forwarded by a sequence of nodes to reach its destination. The mobile network nodes can move arbitrarily, thus the topology is expected to change often and unpredictably. Hence, ad hoc networks require highly adaptive routing protocols and efficient failure recovery strategies to deal with the frequent topology changes. Also, bandwidth and the power of network nodes are limited making protocol development challenging.

Permission to make digital or hard copies of all or part of this work for personal or classroom use is granted without fee provided that copies are not made or distributed for profit or commercial advantage and that copies bear this notice and the full citation on the first page. To copy otherwise, or republish, to post on servers or to redistribute to lists, requires prior specific permission and/or a fee.

MSWIM'04, October 4-6, 2004, Venezia, Italy.

Convright 2004 ACM 1-58113-953-5/04/0010 ..\$5.00.
A mobile ad hoc networking (MANET) working group [1] has been formed within the Internet Engineering Task Force (IETF) to develop protocols including a routing framework for ad hoc networks. A great deal of research has appeared on the development of ad-hoc networks and many routing protocols have been proposed to solve the dynamic multihop routing problem [17]. While significant progress has been made towards developing mobile ad-hoc networks, relatively little work has appeared developing analytical based performance models of mobile ad-hoc networks. Traditionally, mobile ad-hoc network performance has been evaluated using discrete event simulation. The basic simulation approach adopted in the majority of the literature (e.g., $[2],[6],[7])$ is for a given scenario (i.e., number of nodes, mobility model, transmission range, routing scheme, etc.) the network is simulated over a fixed time period (e.g., $600 \mathrm{sec}$ in [2]), multiple runs are made with different random number seeds and the collected data is averaged over the runs. In terms of simulation methodology this approach is considered a steady-state simulation ([8], [9]) and it is recommended that the statistics be cleared after the initial transient in each runs to avoid initialization bias. Analysis of the simulation result takes the form of confidence intervals on the performance metrics. Typical performance metrics are the mean delay, packet delivery fraction, and mean hop count. The simulation program must model the network topology, network traffic and protocols, and unique ad-hoc features such as node mobility, wireless link quality, and limited battery life should be included. Due to the complexity in the simulation modeling of mobile ad-hoc networks, special simulation environments have been developed for their study, such as GloMoSim, Qualnet and ns-2 with CMU extensions. Even with the advent of these specialized simulation tools, the scalability of ad-hoc network simulation for performance studies is an issue.

A weakness of most of the literature on the performance of mobile ad-hoc networks is that steady state analysis techniques are used even though transient or nonstationary periods will occur in the network, especially after a link or node failure. The importance of this transient behavior after failures has been illustrated in several wired network technologies including circuit switched [10] networks, packet switched data networks [11] and in packet based signaling networks [12]. This work taken together shows that the dominant factor on network performance after a failure is this transient or nonstationary congestion period. Due to the rapidly changing topology of mobile ad-hoc networks, one would expect that transient/nonstationary conditions to occur often and possibly dominant the performance behavior. Hence, routing, QoS and congestion techniques designed and evaluated via steady state 
analysis may not make optimum use of network resources after a failure. For example, we have recently studied via simulation the transient behavior of the mobile ad-hoc network Dynamic Source Routing (DSR) protocol in case of path failures and presented an improvement scheme called Failure Record [13]. Specifically, we observed that in a single frequency channel ad hoc network, path failures often cause packets en route to block the interface queue at the upstream node of a failed link. This queue blocking will decrease the performance of the network. The Failure Record scheme prevents such packets from blocking the queue by making every node have a short "memory" of the past and drop the packets before they enter the queue. Simulation results showed that our scheme can significantly improve the performance mean delay and packet delivery fraction of DSR.

While simulation of the time varying/nonstationary behavior of mobile ad-hoc networks is possible, it is computationally difficult. Specifically, to capture the dynamic behavior of the network one must perform a simulation study according to the nonstationary simulation methodology described in [14]. The basic approach is to observe the system behavior versus time over an ensemble of statistically identical but distinct independent runs. The quantities of interest (e.g. mean queue length at every node) are averaged across the ensemble of runs at a particular time instant and confidence intervals can be calculated from the ensemble. Many such points may be obtained at different time instants and the behavior of the system can be determined as a function of time. The principle difficulty in conducting simulation studies of this type is the large number of runs (e.g., thousands) that must be generated in order to get a representative ensemble from which a statistically accurate portrayal of the system behavior can be determined. Hence, very large amounts of CPU time are required for even moderate wired networks and this approach is quite difficult to scale.

In this paper we develop a performance modeling technique for analyzing the time varying performance of mobile ad-hoc networks. Our approach is a novel hybrid of discrete event simulation and numerical methods based queueing analysis. Network queues are modeled using fluid-flow based differential equation models which are solved using numerical methods, while node mobility is modeled using discrete event simulation techniques. Such an approach is more scalable than a nonstationary simulation and allows the modeler insight into the interaction of network nodes/protocols. The rest of the paper is organized as follows. Section 2 provides the details of our modeling approach. Section 3, shows sample numerical results. Conclusions and our future work are given in section 4 .

\section{Modeling Time Varying Behavior}

Consider an ad-hoc network consisting of $M$ nodes which move in an arbitrary fashion. In order to study the dynamic network performance we need to determine the time varying behavior of the queueing model of the network. Note that the main effect of node mobility is to dynamically change the topology of the queueing network model. Determining closed form expressions for the general time varying behavior of such a dynamic topology queueing network is extremely difficult if not impossible $[15,16$, 19]. Here we propose a novel hybrid approach to approximate the network performance using two components, namely: (1) an adjacency matrix model of the network topology which models node movement and (2) a fluid flow model based set of differential equations modeling the time varying queueing behavior at each node. We discuss each component in turn below.

\subsection{Mobility Modeling}

Unlike cellular network mobility, both ends of a wireless link in a mobile ad hoc network are free to move. We model the impact of node mobility and the resulting link failures and additions by representing the network topology at any point in time with a $M \times$ $M$ adjacency matrix denoted by $A(t)$. The matrix is as follows:

$$
A(t)=\left[\begin{array}{cccc}
a_{11}(t) & a_{12}(t) & \cdots & a_{1 M}(t) \\
a_{21}(t) & a_{22}(t) & \cdots & a_{2 M}(t) \\
\vdots & \vdots & & \vdots \\
a_{M 1}(t) & a_{M 1}(t) & \cdots & a_{M M}(t)
\end{array}\right]
$$

where,

$$
a_{i j}(t)=\left\{\begin{array}{l}
1, \quad \text { if node } i \text { and } j \text { are directly connected at time } t \\
0, \quad \text { otherwise }
\end{array}\right.
$$

The connectivity between two nodes is determined by their radio range which is a function of the antenna pattern, power level, geographic terrain, etc., for simplicity we make the common assumption that every node has the same radio range $R$. To determine the adjacency matrix, the distance $d_{i j}$ between every two nodes, $i$ and $j$, is calculated and if the distance is less than or equal to $\mathrm{R}$ then the nodes are connected. Thus,

$$
a_{i j}(t)= \begin{cases}1, & d_{i j} \leq R \quad \text { at time } t \\ 0, & \text { otherwise }\end{cases}
$$

Given an initial placement of the network nodes, the dynamic network topology due to node mobility is reflected in the adjacency matrix by changes in the $a_{i j}(t)$ values with time. The topology change is based on a series of events, which are, either a link breaking and being removed from the topology or a link being added to the topology as two nodes come within radio range. We calculate the time of such events based on the mobility model. Specifically, every node pair is checked for the possible connectivity change based on their current speeds and directions. Note, that changes in speed and direction are also considered events. The event times are placed in chronological order and as time evolves the pair wise connectivity calculation is repeated for every event time and the matrix is changed accordingly. In this way, the adjacency matrix can reflect the topology change dynamically. It is worth noting, that a computationally simpler approach based on direct manipulation of the elements of the adjacency matrix is advocated in [21], where the elements of the adjacency matrix are changed ( 0 or 1$)$ according to a probabilistic model (specifically a two-state MMPP). This approach was shown to be more computationally efficient than detailed simulation of node movement, while giving similar results to the random waypoint mobility model in terms of the network topology changes. Note that either approach can be integrated into the model proposed here. 


\subsection{Node Queueing Model}

As noted above determining the exact time dependent behavior of a queueing system under nonstationary conditions is difficult [16, 17]. Here we adopt a fluid-flow based approximation technique to determine the time varying behavior of the queue at each network node. Specifically, we use the Pointwise Stationary Fluid Flow Approximation (PSFFA) method discussed in [15, 17]. The PSFFA method models the average number in the system at a queue by one or more differential equations which is solved numerically. The use of the PSFFA approach to determine the nonstationary behavior of general finite and infinite capacity queueing systems is discussed in detail in $[15,17]$ and is shown to be quite accurate when compared to nonstationary simulations for a wide variety of cases. Here we derive the PSFFA model for a multi-traffic class $\mathrm{M} / \mathrm{M} / 1$ queueing model of an ad-hoc network node and show how it is linked to the mobility model

\subsubsection{Fluid Flow Background}

Consider a single server queueing system with a nonstationary arrival process. Let $\mu$ denote the average queue service rate and $\lambda(\mathrm{t})$ denote the ensemble average arrival rate at time $\mathrm{t}$ We define $x(t)$ as the state variable representing the ensemble average number in the system at time $t$. Let $\dot{x}(t)$ be the rate of change of the state variable with respect to time. From the flow conservation principle, the rate of change of the average number in the system is equal to the difference between the average arrival and departure rates. Let $f_{\text {in }}(t)$ and $f_{\text {out }}(t)$ denote the ensemble average flow in and out of the system at time $t$, respectively. Then the rate of change of the state variable can be related to the flow in and flow out by

$$
\dot{x}(t)=-f_{\text {out }}(t)+f_{\text {in }}(t)
$$

This type of equation can be found in several places in the literature and is commonly referred to as a fluid flow or dynamic flow equation [15, 17-19]. The flow out of the system can be related to the ensemble average utilization of the server $\rho(t)$ by $f_{\text {out }}(t)=\mu \rho(t)$. If the queue waiting space is large enough to be considered infinite, then the flow into the system is just the arrival rate (i.e., $f_{\text {in }}(t)=\lambda(\mathrm{t})$ ) and the fluid flow model of equation (1) becomes

$$
\dot{x}(t)=-\mu \rho(t)+\lambda(t)
$$

The expression for $\rho(t)$ in equation (2) will depend on the queueing system under study. In general, determining an exact expression for $\rho(t)$ is quite difficult even for the simplest queues. Hence, an approximate method is adopted. The general idea is to determine the values for $\rho(t)$ at particular instants of time by a pointwise mapping from the current value of $x(t)$ into $\rho$ using the steady state queueing relationships. Then the value of $\rho$ thus obtained is used to numerically solve (2) over a small time interval to get a new $x(t)$ and the procedure is repeated for the next time step. Considering the infinite queue case of equation (2), we assume that at steady state (i.e., $x(t)=0$ ) the following functional relationship can be determined:

$$
x=G(\rho)
$$

Additionally, we assume that the functional relationship $G(\rho)$ is numerically invertible, that is $\rho=G^{-1}(x)$. This results in the PSFFA model

$$
\dot{x}(t)=-\mu G^{-1}(x(t))+\lambda(t)
$$

Note that equation (4) is quite general in nature, the only requirement being that the functional relationship $G$ be determined and invertible. For many queueing systems the function $G$ is well known in closed form. Furthermore, for some queueing systems $G$ is invertible and one can derive a closed form expression for the PSFFA model as given in [17]. This is however not a requirement, as the function $G$ can be determined numerically or by curve fitting from measurements for an existing system. One advantage of determining the approximate expression for $\rho(t)$ in (2) using the approach above is that the resulting fluid flow model (4) is exact under steady state conditions. Hence, in solution of the PSFFA model for the time varying response, the model will always converge to the correct steady state value.

Standard numerical integration techniques such as Runge Kutta [20] can be iteratively applied to solve equation (4). The basic solution procedure is described here. We identify the initial condition for the state variable at time zero as $x(0)$ and assume the arrival rate to be a constant over a very small time step $[0, \Delta t]$ (i.e., $\lambda(t)=\lambda(\Delta t / 2)$ for $t \in[0, \Delta t])$. Then equation (4) can be numerically integrated for the value of the state variable at the end of the time interval, $x(\Delta t)$. Note that in solving the fluid flow model over a small time interval one may need to apply a numerical procedure to find $G^{-1}(x)$. The state variable value at the end of the time interval, $x(\Delta t)$, then becomes the initial condition for the next time step $[\Delta t, 2 \Delta t]$. We then adjust the arrival rate for the new time step. This procedure is repeated for each time interval in the time horizon.

\subsubsection{Multi-traffic class M/M/1 fluid flow model}

To apply the fluid flow modeling approach to ad-hoc networks, we first develop the $\mathrm{M} / \mathrm{M} / 1$ queue fluid flow model and its extension to the multi-traffic class case as in [15]. We make the standard product form queueing network assumptions, which allows the queue at each network node to be modeled as a $\mathrm{M} / \mathrm{M} / 1$ type. Specifically, we define $C$ as the wireless link capacity, the packets are assumed exponentially distributed in length with mean length $1 / \mu$, packet transmission times are proportional to the packet length and traffic is assumed to arrive to the queue according to a nonstationary Poisson process with time varying mean rate $\lambda(\mathrm{t})$. For the $\mathrm{M} / \mathrm{M} / 1$ queue at steady state, one gets $x=\lambda /(\mu C-\lambda)$ which corresponds to the functional relationship $x=G(\rho)$ of equation (3) and in this case it can be inverted in closed form to yield the PSFFA model from equation (4) as

$$
\dot{x}(t)=-\mu C\left(\frac{x(t)}{1+x(t)}\right)+\lambda(t)
$$

The accuracy of this model has been studied in $[15,18]$ and an alternative derivation directly from the Chapman-Kolmogorov differential equation model of an $\mathrm{M} / \mathrm{M} / 1$ queue is given in [22]. In ad-hoc networks, the traffic in the network is normally divided into a number of classes and the control actions (i.e. routing and flow control) are based on the class type. Thus the fluid flow model must 
be modified to represent the dynamic behavior of each class separately. Consider an arbitrary network node as shown in Figure 1.

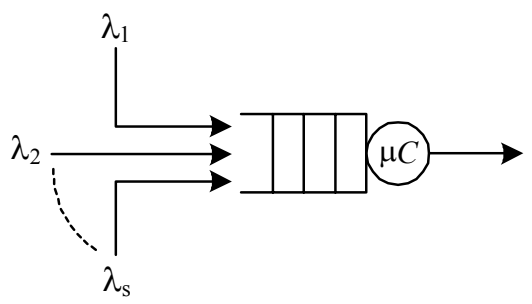

Figure 1. Model of a node with $S$ classes of traffic

There are $S$ different classes of packets arriving at the node with the average arrival rates $\lambda_{I}(t), \lambda_{2}(t), \ldots, \lambda_{S}(t)$. Let $x_{l}(t)$ represent the average number of packets of class $l$ in the queueing system at time $t$. The fluid flow model (5) describing the average total number of packets at the link at time $t, x_{T}(t)$ where $x_{T}=\sum_{l=1}^{S} x_{l}(t)$ is given by

$$
\dot{x}_{T}(t)=-\mu C\left(\frac{x_{T}(t)}{1+x_{T}(t)}\right)+\sum_{l=1}^{S} \lambda_{l}(t)
$$

A fluid flow model of the form of equation (4) can be developed for each class as

$$
\dot{x}_{l}(t)=-\mu C G_{l}^{-1}\left(x_{l}, x_{T}\right)+\lambda_{l}(t) \quad \forall l=1,2, \ldots S
$$

where $G_{l}\left(x_{l}, x_{T}\right)$ represents the average utilization of the link by the class $l$ traffic. Note, that if there are only class $l$ packets in the link then $G_{l}\left(x_{l}, x_{T}\right)$, will be a function of the class $l$ packets $x_{l}$ only and must have the form of the utilization function in model (5) (i.e., $\left.G_{l}\left(x_{l}, x_{T}\right)=x_{l} /\left(1+x_{l}\right)\right)$, since it will represent the dynamics of an $\mathrm{M} / \mathrm{M} / 1$ queue with only one class of traffic. However, if additional classes of traffic are also present in the link, they will use part of the transmission capacity of the link and the portion of link capacity seen by the class $l$ packets will depend on the total amount of link capacity being used. Thus $G_{l}\left(x_{l}, x_{T}\right)$ will be a function of both the average number of class $l$ packets in the link queue $x_{l}$ and the total average number of packets in the link $x_{T}$. We determine $G_{l}\left(x_{l}, x_{T}\right)$ using the PSFFA technique of matching the steady-state equilibrium point of the fluid flow model and the equivalent queueing model. Thus from the $M / M / 1$ queueing model of the node with $\mathrm{S}$ classes of customers, we require at steady-state.

$$
x_{l}=\frac{\lambda_{l}}{\mu C-\sum_{j=1}^{S} \lambda_{j}} \quad \forall l=1,2, \cdots S
$$

Now, at steady-state $\dot{x}_{l}(t)=0, \rightarrow \mu C G^{-1}\left(x_{l}, x_{T}\right)=\lambda_{l} \quad \forall l$ and $\dot{x}_{T}(t)=0, \rightarrow \mu C\left(\frac{x_{T}}{1+x_{T}}\right)=\sum_{j=1}^{S} \lambda_{j}$. Hence, substituting in (8) and solving for $G_{l}^{-1}\left(x_{l}, x_{T}\right)$, results in

$$
G_{l}^{-1}\left(x_{l}, x_{T}\right)=\left(\frac{x_{l}}{1+x_{T}}\right)=\left(\frac{x_{l}}{1+\sum_{j=1}^{S} x_{j}}\right) \forall l
$$

and the fluid flow model for the node now becomes

$$
\dot{x}_{l}(t)=-\mu C\left(\frac{x_{l}(t)}{1+\sum_{j=1}^{S} x_{j}(t)}\right)+\lambda_{l}(t) \quad \forall l=1,2, \cdots S
$$

Thus, a node can be described by a set of $S$ nonlinear differential equations of the form of equation (9) representing the time varying behavior of each traffic class separately. The system of equations can be solved using the numerical integration approach discussed above and results on the accuracy and computational advantages of this type of model in comparison to simulation are given in [15]. The multi-traffic class fluid flow model developed above represents the dynamics of a single isolated node in a network and we now extend the model to represent the ad-hoc network environment.

Thus, a node can be described by a set of $S$ nonlinear differential equations of the form of equation (9) representing the time varying behavior of each traffic class separately. The system of equations can be solved using the numerical integration approach discussed above and results on the accuracy and computational advantages of this type of model in comparison to simulation are given in [15]. The multi-traffic class fluid flow model developed above represents the dynamics of a single isolated node in a network and we now extend the model to represent the ad-hoc network environment.

\subsubsection{A Fluid Flow Model of an Ad-hoc Network}

Consider an ad hoc network consisting of M nodes, an arbitrary node $i$ is shown in Figure 2 below. At each node the packets are typed into classes based on their destination node, thus there are $M-1$ possible traffic classes at each node. We assume that packets are generated at the network node $i$ destined for node $j$ according to a Poisson process (which can be nonstationary), with mean rate $\gamma_{i}^{j}(t)$. We define $\gamma_{i}(t)$ as the vector of all mean traffic rates generated at node $i, \gamma_{i}(t)=\left[\gamma_{i}^{j}(t), \forall j, j \neq i\right]$. Similarly, we define $x_{i}^{j}(\mathrm{t})$ as the mean number of packets in the queueing system at node $i$ destined for node $j$ at time $t$ and $x_{i}(t)$ as a row vector of state variables at node $i$ where, $x_{i}(t)=\left[x_{i}^{j}(t), \forall j, j \neq i\right]$. We assume exponentially distributed packet lengths with mean packet length $\mu$ and let $C_{i}$ denote the transmission capacity of node $i$. Given these assumptions the multi-class fluid flow model of (9) would apply if there the node was not being used as a relay for other traffic, had only one out going link and was not moving. In order to include mobility, the possibility of serving as a relay node and multiple outgoing links, we must incorporate routing variables and the dynamic nature of the topology. We define $r_{i k}^{j}(t)$ as a routing variable from node $i$ to node $k$ for traffic destined for node $j$. The value of the routing variable at any point in time depends on the specific routing scheme used (e.g., flooding, DSR, etc.) and the current network topology. As described in section 2.1, $a_{i k}(t)$ denotes a zero/one indicator variable that is equal to one if node $i$ and $k$ are within the radio range of each other,.

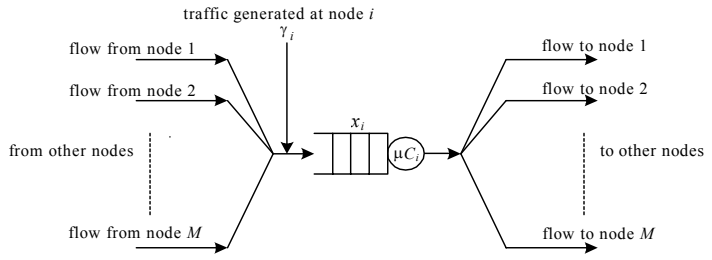

Figure 2. Arbitrary node $i$ queueing model 
Note that the flow out of node $i$ to node $k$ of a particular traffic class $j$ will depend upon the existence of a direct link between $i$ and $k$ and the routing variables for traffic class $j$. Hence one must modify the flow out term in (9) to incorporate $a_{i k}(t)$ and $r_{i k}^{j}(t)$. Specifically the flow out of node $i$ of class $j$ traffic to node $k$ is given by

$$
=\mu C_{i}\left(\frac{x_{i}^{j}(t)}{1+\sum_{\substack{l=1 \\ l \neq i}}^{M} x_{i}^{l}(t)}\right)\left(a_{i k}(t) r_{i k}^{j}(t)\right)
$$$$
\text { class } j \text { traffic flow out of node i to node } k
$$

The flow of class $j$ traffic into the node $i$ queue will consist of traffic generated at node $i$ with rate $\gamma_{i}^{j}(t)$ and the flow of class $j$ traffic to node $i$ from other network nodes. Specifically, the flow of class $j$ traffic into node $i$ from node $l$ is given by.

$$
=\mu C_{l}\left(\frac{x_{l}^{j}(t)}{1+\sum_{\substack{k=1 \\ k \neq l}}^{M} x_{l}^{k}(t)}\right)\left(a_{l i}(t) r_{l i}^{j}(t)\right)
$$$$
\text { class } j \text { traffic flow in to node } i \text { from node } l
$$

The resulting ad-hoc network fluid-flow model is determined by summing the flow in and out over all possible nodes and is given by

$$
\dot{x}_{i}^{j}(t)=-\mu C_{i}\left(\frac{x_{i}^{j}(t)}{1+\sum_{\substack{l=1 \\ l \neq i}}^{M} x_{i}^{l}(t)}\right)\left(\sum_{\substack{k=1 \\ k \neq i}}^{M} a_{i k}(t) r_{i k}^{j}\right)+\gamma_{i}^{j}(t)+\sum_{\substack{l=1 \\ l \neq i \\ l \neq j}}^{M} \mu C_{l}\left(\frac{x_{l}^{j}(t)}{1+\sum_{\substack{k=1 \\ k \neq l}}^{M} x_{l}^{k}(t)}\right)\left(a_{l i}(t) r_{l i}^{k}\right)
$$

$\forall i, j=1,2, \ldots M(10)$

In (10) the first term to the right of the equal sign represents the flow out of node $i$ of traffic class $j$, the second term represents the type $j$ traffic generated at node $i$, and the last term denotes the flow of type $j$ traffic into node $i$ from other network nodes. Note the two rightmost terms in (10) represent the total class $j$ traffic flow into the queue at node $i$, namely $\lambda_{i}^{j}(t)$. From (10), one can see that a node $i$ is represented by a set of $M-1$ differential equations and the network as a whole by $M \times M-1$ equations. Numerical solution of the model follows a hybrid approach, where the node mobility and adjacency matrix are determined via discrete event simulation and the fluid flow model is solved via numerical integration as discussed above for the PSFFA approach.

\section{NUMERICAL RESULTS}

Here we present preliminary numerical results illustrating the application of the model presented in Section 2. For the sake of simplicity we model a three node network as shown in Figure 3.

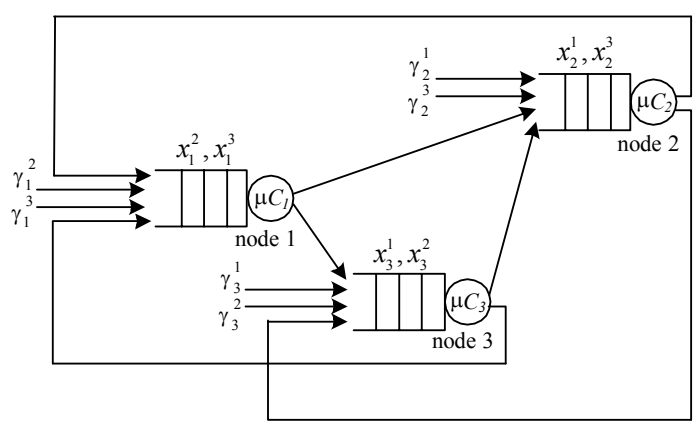

Figure 3. Three node ad-hoc network queueing model

For the network shown in Figure 3, the fluid flow model of (10) results in six differential equations. In the following, we first report baseline results where node movement occurs in a predetermined fashion. Then we examine the results when the link utilization is increased, the node mobility is increased, and the load is changing. In our numerical results we assume $\mathrm{C}_{1}=\mathrm{C}_{2}=\mathrm{C}_{3}$ $=20$, and $\mu=0.05$, which corresponds to a normalized server capacity of one packet per second. For all numerical solutions to the differential equations, the fifth order Runge-Kutta routine provided in MATLAB was utilized. In the numerical solution various values for the time step $\Delta t$ (e.g., $\Delta t=1, \Delta t=0.1$, etc.) over which each integration is conducted were tried, until decreasing the time step resulted in no change in the numerical values.

The topologies illustrated in Figure 4 model a set of the connectivity changes between the three nodes in terms of time that corresponds to a set of node movements.

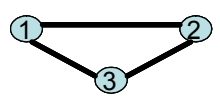

(a) $t<100$

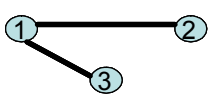

(d) $300<=$ t $<400$

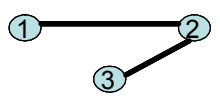

(b) $100<=$ t $<200$

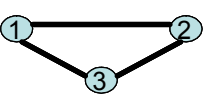

(e) $400<=$ t $<500$

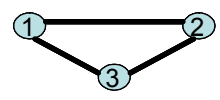

(c) $200<=$ t $<300$

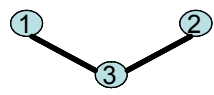

(f) $500<=\mathrm{t}$
Figure 4. A mobility and connectivity scenario

The topologies of Figure 4 (a-f) determine the adjacency matrix values at the corresponding time points. Given a mobility scenario such as above, one can calculate the routing variables $r_{i k}^{j}(t)$ using a specific routing algorithm. For example, here we use minimum hop routing. We assume that rerouting of traffic after a topology change (link addition or deletion) occurs instantly, which is a best case scenario. Figure 5 shows the queueing behavior for the two traffic classes at each of the three nodes when the load is $\gamma_{1}^{2}(\mathrm{t})=$ $0.18, \gamma_{1}^{3}(\mathrm{t})=0.22, \gamma_{2}{ }^{1}(\mathrm{t})=0.18, \gamma_{2}^{3}(\mathrm{t})=0.22, \gamma_{3}{ }^{1}(\mathrm{t})=0.18$, and $\gamma_{3}^{2}(\mathrm{t})=0.22$. From Figure 5, one can see the effect of the topology changes on the mean number of packets of each traffic class at each node. For time $\mathrm{t}<100$, all three nodes have gone through an initial transient and reached steady state. At time $\mathrm{t}=$ 100, the link between 1 and 3 breaks and the traffic over it is rerouted through node 2 causing a large transient spike in the 
number in the system until the link between 1 and 3 is restored at time $\mathrm{t}=200$. At time $\mathrm{t}=300$, the link between 3 and 2 breaks resulting in traffic being rerouted through node 1 and large transients there until the link is restored at time $t=400$, Similarly, a large increase in the number in the system occurs at node 3 when the link between 1 and 2 breaks at time $t=500$. Notice, that transient increase is larger at nodes with greater traffic load.

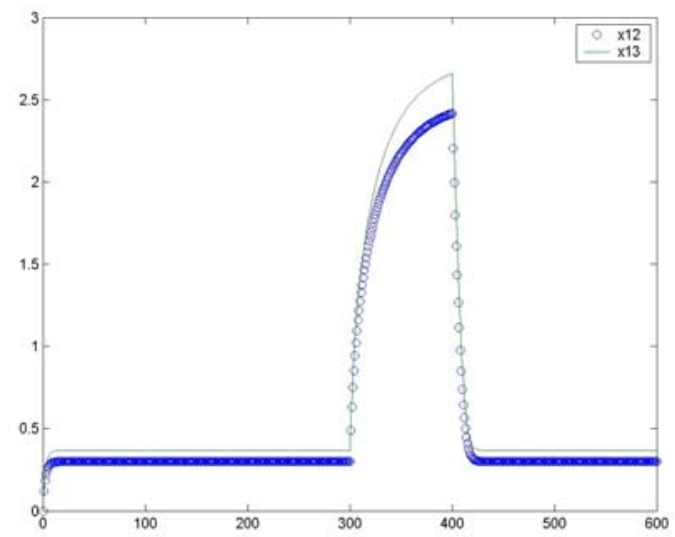

Figure 5 (a). Node 1, Buffers $x_{1}{ }^{2}, x_{1}{ }^{3}$

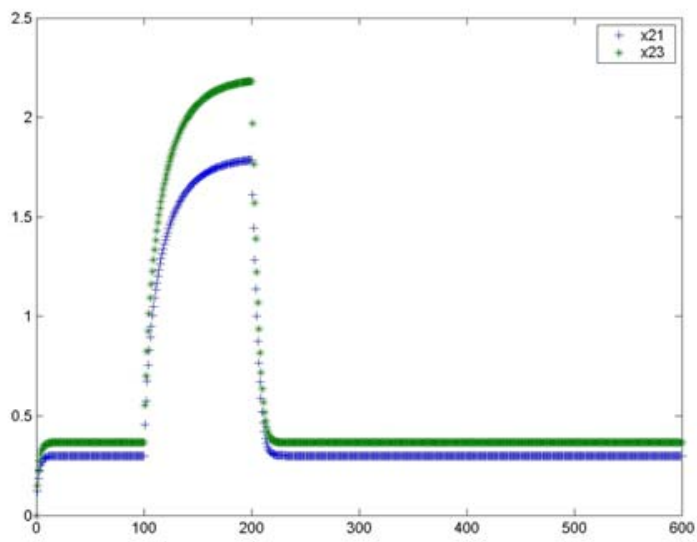

Figure 5 (b). Node 2, Buffers $x_{2}{ }^{1}, x_{2}{ }^{3}$

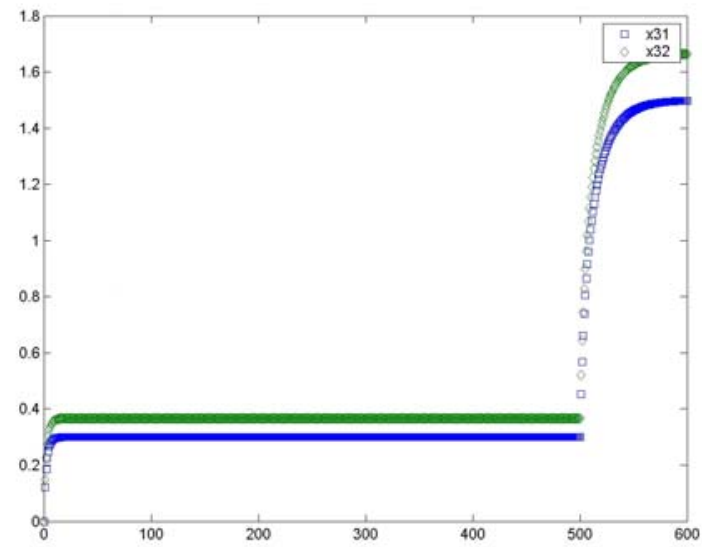

Figure 5 (c). Node 3, Buffers $x_{3}{ }^{1}, x_{3}{ }^{2}$
Next we consider the effect of increasing the load, specifically, the link utilization is increased to 0.6, whereas the results of Figure 5 have a link utilization of 0.4. Figure 6 shows the behavior for each of the two traffic classes at each node when the load is $\gamma_{1}^{2}(t)$ $=0.25, \gamma_{1}{ }^{3}(\mathrm{t})=0.35, \gamma_{2}{ }^{1}(\mathrm{t})=0.35, \gamma_{2}{ }^{3}(\mathrm{t})=0.25, \gamma_{3}{ }^{1}(\mathrm{t})=0.30$, and $\gamma_{3}{ }^{2}(\mathrm{t})=0.30$. We assume the same mobility and connectivity model as described by Figure 4. As one can see from Figure 6, the behavior is similar to those of Figure 5, except that, the number in system has increased by about 7 times.

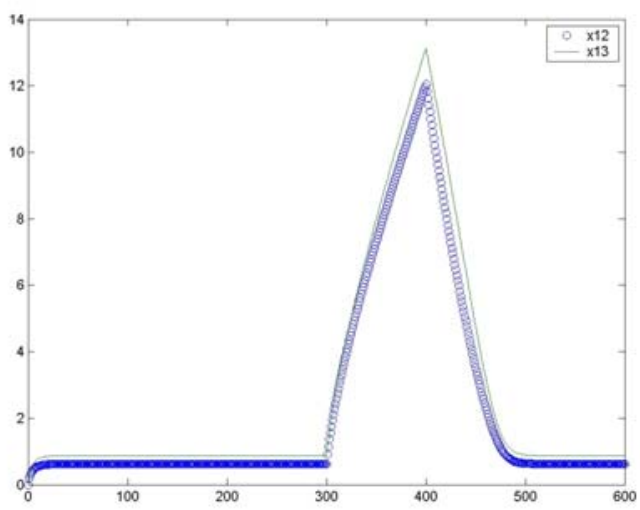

Figure 6 (a). Node 1, Buffers $x_{1}{ }^{2}, x_{1}{ }^{3}$

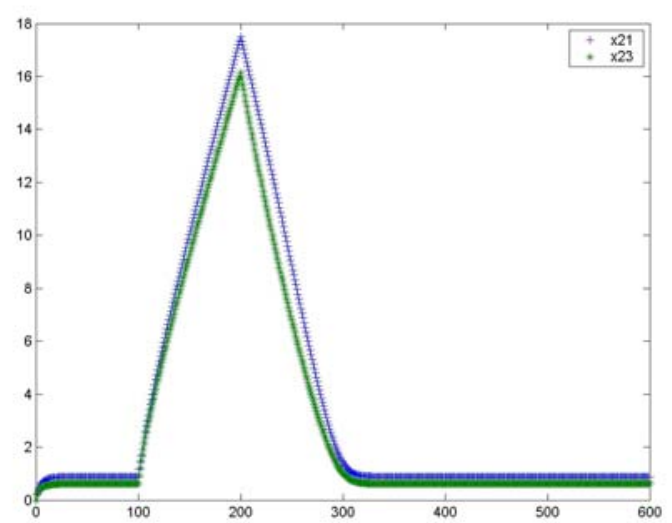

Figure 6 (b). Node 2, Buffers $x_{2}{ }^{1}, x_{2}{ }^{3}$

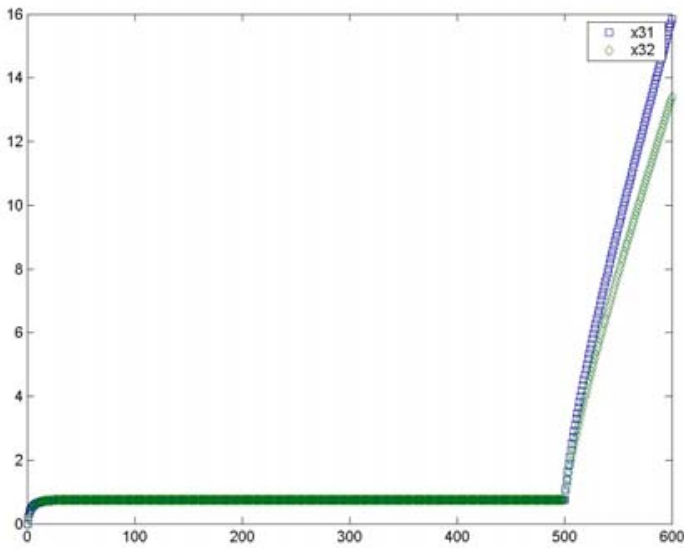

Figure 6 (c). Node 3, Buffers $x_{3}{ }^{1}, x_{3}{ }^{2}$ 
When the node mobility is increased, topology changes occur more frequently. Here we consider the topology changes as shown in Figure 7. Figure 8 shows the behavior for the two traffic classes at each of the nodes when the load is the same as that used for the baseline case of Figure 5. Figure 9 shows the buffer behavior for the two traffic classes at each of the three nodes when the load is increased to the same as that used for generating Figure 6 .

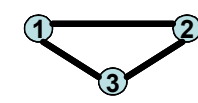

(a) $\mathrm{t}<20$ or $200<=\mathrm{t}<210$, or $300<=\mathrm{t}<320$, (1) or $500<=\mathrm{t}<510$ 3

(d) $110<=$ t $<150$ or $260<=\mathrm{t}<280$, or $410<=\mathrm{t}<450$, or $560<=\mathrm{t}<580$

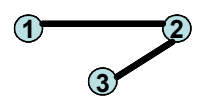

(b) $20<=$ t $<100$, or $210<=\mathrm{t}<250$, or $320<=\mathrm{t}<400$, or $510<=\mathrm{t}<550$
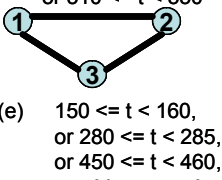

or $580<=\mathrm{t}<585$

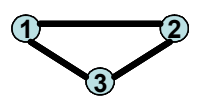

(c) $100<=\mathrm{t}<110$ or $400<=\mathrm{t}<410$ or $550<=\mathrm{t}<560$

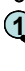
or $285<=\mathrm{t}<300$ or $460<=\mathrm{t}<500$ or $585<=\mathrm{t}$ or $250<=\mathrm{t}<260$

(f) $160<=\mathrm{t}<200$,

Figure 7. Rapid mobility and connectivity scenario

As one can be seen from Figure 8, when the topology changes more rapidly, significant transient/nonstationary periods occur even in a lightly loaded network. From Figure 9, it is observed that when the network is more heavily loaded the transient effects are even more pronounced.

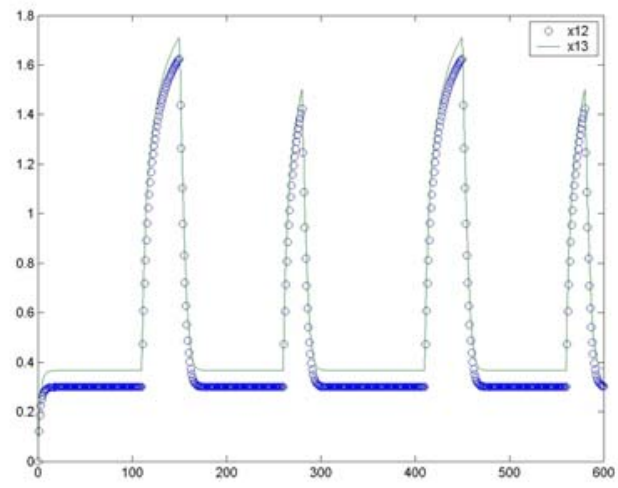

Figure 8 (a). Node 1, Buffers $x_{1}{ }^{2}, x_{1}{ }^{3}$

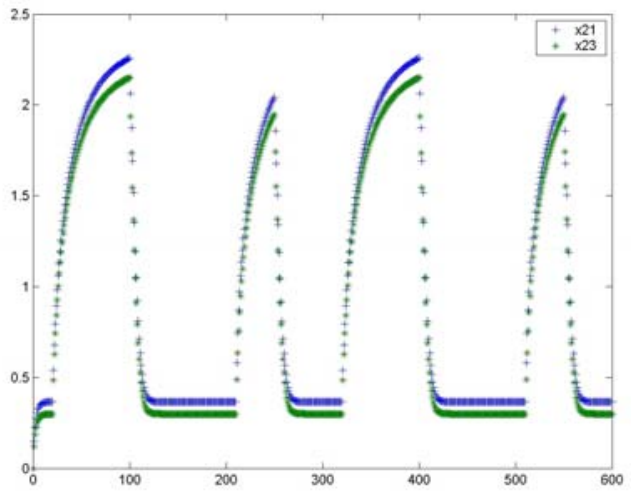

Figure 8 (b). Node 2, Buffers $x_{2}{ }^{1}, x_{2}{ }^{3}$

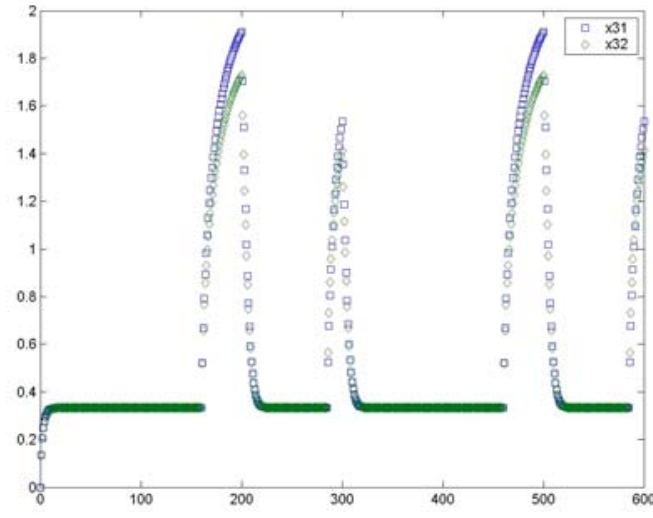

Figure 8 (c). Node 3, Buffers $x_{3}{ }^{1}, x_{3}{ }^{2}$

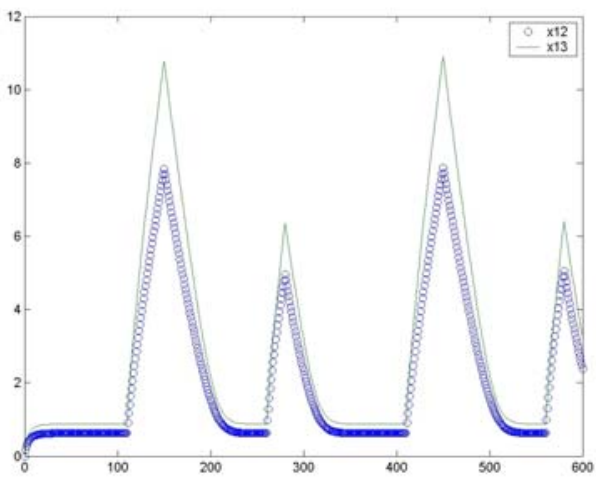

Figure 9 (a). Node 1, Buffers $x_{1}{ }^{2}, x_{1}{ }^{3}$

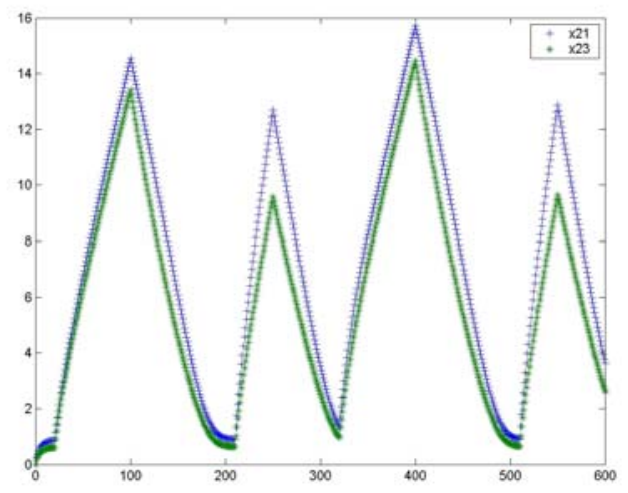

Figure 9(b). Node 2, Buffers $x_{2}{ }^{1}, x_{2}{ }^{3}$ 


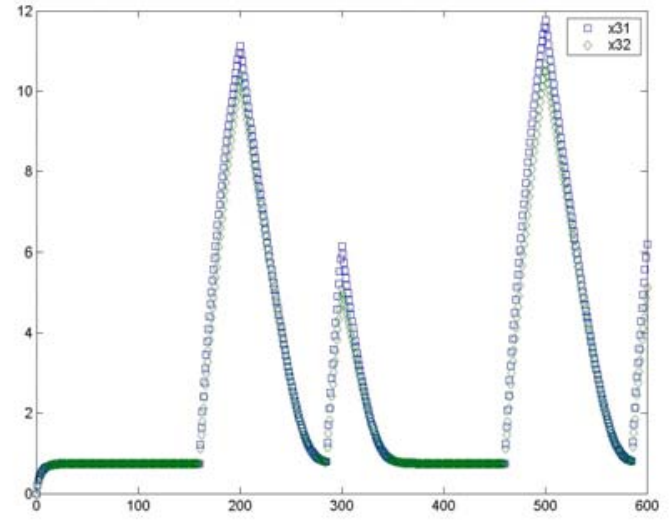

Figure 9 (c). Node 3, Buffers $x_{3}{ }^{1}, x_{3}{ }^{2}$

Next with the same topology changes as described in Figure 4 we randomly vary the load at each node. Specifically, the load at each node $\gamma_{i}^{j}$ is composed of group of ten small flows. The small flows have an exponentially distributed on duration with mean of 10 seconds and a exponentially distributed off time with a mean of 2 seconds. The mean rate of each flow is drawn from a uniform distribution (e.g., $\mathrm{u}[0.03,0.05])$ and the average link utilization is 0.5 . Figure 10 , shows the results for each node. One can see the effect of the load changes and the topology changes on the mean number of packets of each traffic class at each node. Note at time $\mathrm{t}=100$, the link between 1 and 3 breaks and the traffic over it is rerouted through node 2 causing a large transient spike in the number in the system until the link between 1 and 3 is restored at time $\mathrm{t}=200$. Similar behavior is seen at the other nodes.

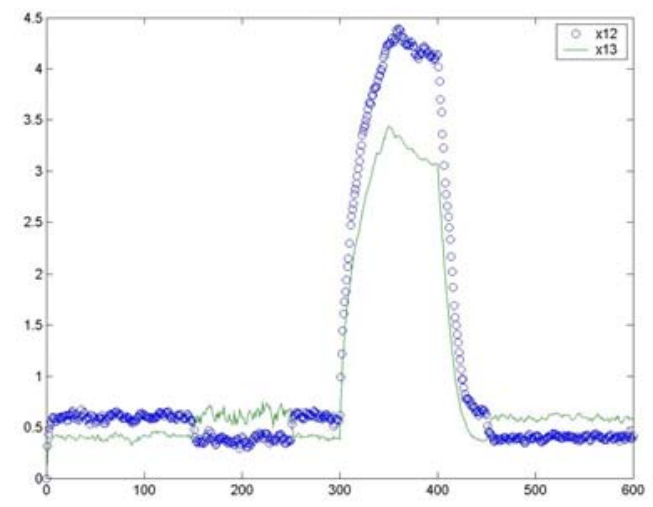

Figure 10 (a). Node 1, Buffers $x_{1}{ }^{2}, x_{1}{ }^{3}$

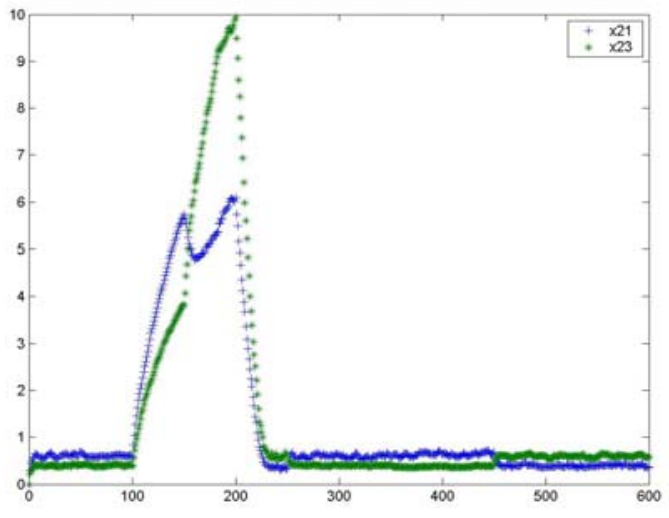

Figure 10 (b). Node 2, Buffers $x_{2}{ }^{1}, x_{2}{ }^{3}$

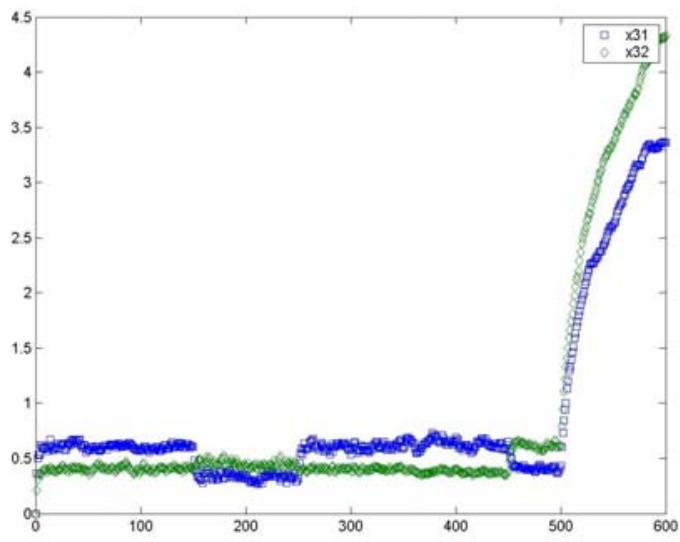

Figure 10 (c). Node 3, Buffers $\mathrm{x}_{3}{ }^{1}, \mathrm{x}_{3}{ }^{2}$

\section{CONCLUSIONS}

In this paper we develop a performance modeling technique for analyzing the time varying behavior of mobile ad hoc networks. Our approach is a novel hybrid of discrete event simulation and numerical method based queueing analysis. Network queues are modeled using fluid-flow based differential equation models which are solved using numerical methods, while node mobility is modeled using an adjacency matrix topology representation whose values are determined via discrete event simulation techniques. Preliminary numerical results were given illustrating the approach for a sample network. Our future work includes determining the accuracy of the approach in comparison with simulation results from ns-2, generating numerical results for a wide variety of parameter values (e.g., different mobility models, different routing schemes) as well as larger networks and examining the scalability of the approach.

\section{REFERENCES}

[1] J. Macker and S. Corson, "Mobile Ad Hoc Networks (MANET)", Internet Engineering Task Force WG Charter. 1997, http://www.ietf.org/html.charters/manetcharter.html,

[2] S. Lee, J. Hsu, R. Hayashida, M. Gerla, R. Bagrodia, "Selecting a Routing Strategy for Your Ad-hoc Network", 
Computer Communications, Vol. 26, pp.723-733, Oct. 2003

[3] C. Perkins, Ad-Hoc Networks, Addison Wesley, 2000

[4] E. M. Royer and C. - K. Toh, "A Review of Current Routing Protocols for Ad Hoc Mobile Wireless Networks", IEEE Personal Communications, April 1999

[5] C. E. Perkins, E. M. Royer and S. R. Das, "Ad-hoc Ondemand Distance Vector (AODV) Routing", IETF Internet Draft, Jan. 2002

[6] C. E. Perkins, E. M. Royer, S. Das, and M. Marina "Performance Comparison of Two On-demand Routing Protocols for Ad Hoc Networks", IEEE Personal Communications, Feb., 2001

[7] T. Camp, J. Boleng, B. Williams, L. Wilcox, W. Navidi, "Performance Comparision of Two Location Based Routing Protocols for Ad Hoc Networks", Proceedings of IEEE INFOCOM 2002, Vol.3, pp.1678-1687, June 2002

[8] K. Pawlikowski, "Steady-State Simulation of Queueing Processes: A Survey of Problems and Solutions", ACM Computing Surveys, Vol. 22., No. 2, pp: 123-170, June, 1990.

[9] J. Banks, J. Carson, B. Nelson, D. Nicol, Discrete Event System Simulation, $3^{\text {rd }}$ ed., Prentice-Hall, 2001

[10] D. Logothetis and K. Trivedi, “'The Effect of Detection and Restoration Times for Error Recovery in Communications Networks", Journal of Network and Systems Management, Vol. 5, pp. 173-196, 1997.

[11] D. Tipper, J. Hammond, S. Sharma, A. Khetan, K. Balakrishnan and S. Menon, "An Analysis of the Congestion Effects of Link Failures in Wide Area Networks", IEEE Journal of Selected Areas in Communications, Vol. 12, pp. 179-192, 1994.

[12] M. Rumsewicz and D. Smith, "A Comparison of SS7 Congestion Control Options During Mass Call-in Situations", IEEE/ACM Transactions on Networking, Feb., 1995.

[13] X. Hou and D. Tipper, "Impact of Failures on Routing in Mobile Ad Hoc Networks Using DSR", Proceedings of Communication Networks and Distributed Systems
Modeling and Simulation Conference (CNDS), Jan., 2003, Orlando, FL

[14] W. Lovegrove, J. Hammond and D. Tipper, "Simulation Methods for Studying Nonstationary Behavior of Computer Networks", IEEE Journal on Selected Areas in Communications, vol. 8, no. 9, pp. 1696-1708, Dec. 1990

[15] D. Tipper and M. K. Sundareshan, Numerical Methods for Modeling Computer Networks Under Nonstationary Conditions", IEEE Journal on Selected Areas in Communications, vol. 8, no. 9, pp. 1682-1695, Dec. 1990

[16] W. Massey, "The Analysis of Queue with Time-Varying Rates for Telecommunication Models", Telecommunication Systems, Vol. 21, pp. 173-204, 2002

[17] W. Wang, D. Tipper and S. Banerjee, "A Simple Approximation for Modeling Nonstationary Queues," Proceedings of IEEE INFOCOM '96, San Francisco, CA, 1996.

[18] J. Filipiak, Modelling and Control of Dynamic Flows in Communication Networks, Springer-Verlag, Berlin, 1988.

[19] X. Gu, K. Sohraby, and D. Vamen, Control and Performance in Packet, Circuit and ATM Networks, Kluwer Academic, Norwell, MA, 1995.

[20] R. Burden, J. Faires, and A. Reynolds, Numerical Analysis, $2^{\text {nd }}$ Ed., Prindile Weber \& Schmit. Boston, MA. 1981

[21] T. Lin and S. Midkiff, " Mobility versus Link Stability in the Simulation of Mobile Ad Hoc Networks Using DSR", Proceedings of Communication Networks and Distributed Systems Modeling and Simulation Conference (CNDS), Jan., 2003, Orlando, FL

[22] K. Rider, "A Simple Approximation to the Average Queue Size in the Time Dependent M/M/1 queue," Journal of $A C M$, Vol. 23, No. 2, pp. 361-367, 1976. 